\title{
EXTREME RANKS OF (SKEW-)HERMITIAN SOLUTIONS TO A QUATERNION MATRIX EQUATION*
}

\author{
QING WEN WANG ${ }^{\dagger}$ AND JING JIANG $\ddagger$
}

\begin{abstract}
The extreme ranks, i.e., the maximal and minimal ranks, are established for the general Hermitian solution as well as the general skew-Hermitian solution to the classical matrix equation $A X A^{*}+B Y B^{*}=C$ over the quaternion algebra. Also given in this paper are the formulas of extreme ranks of real matrices $X_{i}, Y_{i}, i=1, \cdots, 4$, in a pair (skew-)Hermitian solution $X=$ $X_{1}+X_{2} i+X_{3} j+X_{4} k, Y=Y_{1}+Y_{2} i+Y_{3} j+Y_{4} k$. Moreover, the necessary and sufficient conditions for the existence of a real (skew-)symmetric solution, a complex (skew-)Hermitian solution, and a pure imaginary (skew-)Hermitian solution to the matrix equation mentioned above are presented in this paper. Also established are expressions of such solutions to the equation when corresponding solvability conditions are satisfied. The findings of this paper widely extend the known results in the literature.
\end{abstract}

Key words. Quaternion matrix equation, Minimal rank, Maximal rank, Hermitian solution, Skew-Hermitian solution.

AMS subject classifications. 15A03, 15A09, 15A24, 15A33.

1. Introduction. Throughout this paper, we denote the real number field by $\mathbb{R}$, the complex number field by $\mathbb{C}$, the set of all $m \times n$ matrices over the quaternion algebra

$$
\mathbb{H}=\left\{a_{0}+a_{1} i+a_{2} j+a_{3} k \mid i^{2}=j^{2}=k^{2}=i j k=-1, a_{0}, a_{1}, a_{2}, a_{3} \in \mathbb{R}\right\}
$$

by $\mathbb{H}^{m \times n}$, the identity matrix with the appropriate size by $I$. For a quaternion matrix $A$, we denote the column right space, the row left space of $A$ by $\mathcal{R}(A), \mathcal{N}(A)$, respectively, the dimension of $\mathcal{R}(A)$ by $\operatorname{dim} \mathcal{R}(A)$. By [9], $\operatorname{dim} \mathcal{R}(A)=\operatorname{dim} \mathcal{N}(A)$, which is called the rank of $A$, and denoted by $r(A)$. The Moore-Penrose inverse of a matrix $A \in \mathbb{H}^{m \times n}$, denoted by $A^{\dagger}$, is defined to be the unique matrix $X \in \mathbb{H}^{n \times m}$ satisfying

${ }^{*}$ Received by the editors February 14, 2010. Accepted for publication July 31, 2010. Handling Editor: Michael Neumann.

$\dagger$ Department of Mathematics, Shanghai University, Shanghai 200444, P.R. China (wqw858@yahoo.com.cn). Supported by the grants from the Ph.D. Programs Foundation of Ministry of Education of China (20093108110001), the Scientific Research Innovation Foundation of Shanghai Municipal Education Commission (09YZ13), Natural Science Foundation of China (60672160), Singapore MoE Tier 1 Research Grant RG60/07, and Shanghai Leading Academic Discipline Project (J50101).

${ }^{\ddagger}$ Department of Mathematics, Shanghai University, Shanghai 200444, P.R. China, and Department of Mathematics and Applied Mathematics, QiLu Normal University, Jinan 250013, P.R. China. 
the following four matrix equations

$$
\text { (1) } A X A=A,(2) X A X=X,(3)(A X)^{*}=A X,(4)(X A)^{*}=X A \text {. }
$$

Moreover, $L_{A}$ and $R_{A}$ stand for the two projectors $L_{A}=I-A^{\dagger} A, R_{A}=I-A A^{\dagger}$ induced by $A$.

We know that matrix equation is one of the topics of very active research in matrix theory and applications, and a large number of papers have presented several methods for solving several matrix equations (e.g. [4]-[6], [10], [23]-[26], [29]-[31], [37], [39], [42]). As a very classical linear matrix equation,

$$
A X A^{*}+B Y B^{*}=C
$$

has been investigated by many authors from different aspects. For example, using generalized singular value decomposition, Chang and Wang [2] derived the expressions for the general symmetric and minimum-2-norm symmetric solutions to (1.1) within the real setting. $\mathrm{Xu}$, Wei, and Zheng [38] obtained the general form of all least-squares Hermitian (skew-Hermitian) solutions to (1.1). Liao and Bai [11] used generalized singular value decomposition to investigate the symmetric positive semidefinite solution to (1.1). Zhang [40] gave necessary and sufficient conditions for the existence of a Hermitian nonnegative-definite solution to (1.1). Wang and Zhang [32] gave a necessary and sufficient condition for the existence and an expression for the re-nonnegative definite solution to (1.1) over $\mathbb{H}$ by using the decomposition of pairwise matrices.

Research on extreme ranks, i.e., maximal and minimal ranks, of solutions to linear matrix equations have been actively ongoing for more than 30 years (see [15]-[17], [19], [20], [27], [28], [33]-[35]). It is worthy to say that minimal and maximal ranks of a general solution to a matrix equation are very useful in linear programming computations (see [15]-[17]). In 2009, Liu, Tian and Takane [13] presented formulas for the maximal and minimal ranks of a Hermitian solution and a skew-Hermitian solution to the special case of the matrix equation (1.1) in which $B=0$. The following matrix equation:

$$
A X A^{*}=C
$$

over $\mathbb{C}$, has been well examined by many authors (see [1], [3], [7], [8], [32], [36], [41]).

Note that, to our knowledge, there has been little information on extreme ranks of the (skew-)Hermitian solution to the matrix equation (1.1). This paper aims to consider the formulas of extremal ranks of the general (skew-)Hermitian solution to (1.1).

The paper is organized as follows. In Section 2, we first give an expression of the general Hermitian solution to (1.1) by using the generalized inverses of the co-

efficient matrices of this equation, then derive the formulas of extremal ranks of the 
general Hermitian solution to (1.1). We also give the corresponding results on the skew-Hermitian solution to (1.1). In Section 3, we first present the maximal and minimal ranks of the real matrices $X_{1}, X_{2}, X_{3}, X_{4}$ and $Y_{1}, Y_{2}, Y_{3}, Y_{4}$ in a Hermitian solution $X=X_{1}+X_{2} i+X_{3} j+X_{4} k, Y=Y_{1}+Y_{2} i+Y_{3} j+Y_{4} k$ to (1.1), then give some necessary and sufficient conditions for (1.1) to have a real symmetric solution, a complex Hermitian solution, and a pure imaginary Hermitian solution. The corresponding results on the skew-Hermitian solution to (1.1) are also considered. Some special cases of (1.1) are also considered in Section 4.

2. Ranks of the general Hermitian solution to (1.1). In this section, we consider the maximal and minimal ranks of the general (skew-)Hermitian solution to (1.1) over $\mathbb{H}$.

We begin with the following lemma that is due to Tian [18], and can be generalized to $\mathbb{H}$.

Lemma 2.1. Suppose that the matrix equation

$$
A X B+C Y D=E
$$

is consistent over $\mathbb{H}$, where $X \in \mathbb{H}^{p \times q}, Y \in \mathbb{H}^{s \times t}$ unknown. Then the general solution of (2.1) can be expressed by

$$
\begin{aligned}
& X=X_{0}+S_{1} L_{G} U R_{H} T_{1}+L_{A} V_{1}+V_{2} R_{B}, \\
& Y=Y_{0}-S_{2} L_{G} U R_{H} T_{2}+L_{C} W_{1}+W_{2} R_{D},
\end{aligned}
$$

where $X_{0}$ and $Y_{0}$ are a special pair solution of (2.1),

$$
S_{1}=\left(I_{p}, 0\right), S_{2}=\left(0, I_{s}\right), T_{1}=\left(I_{q}, 0\right)^{*}, T_{2}=\left(0, I_{t}\right)^{*}, G=(A, C), H=\left(\begin{array}{c}
B \\
D
\end{array}\right) ;
$$

$U, V_{1}, V_{2}, W_{1}$, and $W_{2}$ are arbitrary quaternion matrices with suitable sizes.

Lemma 2.2. Consider the matrix equation (1.1) where $A \in \mathbb{H}^{m \times n}, B \in \mathbb{H}^{m \times p}$, $C \in \mathbb{H}^{m \times m}$ are given, and $X \in \mathbb{H}^{n \times n}, Y \in \mathbb{H}^{p \times p}$ unknown.

1. If $C=C^{*}$, and (1.1) has a Hermitian solution, then the general Hermitian solution to (1.1) can be expressed as

$$
\begin{aligned}
& X=X_{0}+S_{1} L_{G} Z L_{G} S_{1}^{*}+L_{A} V+V^{*} L_{A}, \\
& Y=Y_{0}-S_{2} L_{G} Z L_{G} S_{2}^{*}+L_{B} W+W^{*} L_{B},
\end{aligned}
$$

where $X_{0}$ and $Y_{0}$ are a special pair Hermitian solution of (1.1),

$$
S_{1}=\left(I_{n}, 0\right), S_{2}=\left(0, I_{p}\right), G=(A, B) ;
$$

$Z$ is an arbitrary Hermitian quaternion matrix with consistent size, and $V$ and $W$ are arbitrary quaternion matrices with suitable sizes. 
2. If $C=-C^{*}$, and (1.1) has a skew-Hermitian solution, then the general skewHermitian solution can be expressed as

$$
\begin{aligned}
& X=X_{0}+S_{1} L_{G} Z L_{G} S_{1}^{*}+L_{A} V-V^{*} L_{A}, \\
& Y=Y_{0}-S_{2} L_{G} Z L_{G} S_{2}^{*}+L_{B} W-W^{*} L_{B},
\end{aligned}
$$

where $X_{0}$ and $Y_{0}$ are a special pair skew-Hermitian solution of (1.1), and $S_{1}$, $S_{2}$, and $G$ are the same as (2.3); $Z$ is an arbitrary skew-Hermitian quaternion matrix with consistent size, and $V$ and $W$ are arbitrary quaternion matrices with suitable sizes.

Proof. We here only prove 1 . The proof of 2 can be similarly finished.

Suppose that $X_{1}=X_{1}^{*}, Y_{1}=Y_{1}^{*}$ are an arbitrary pair Hermitian solution of (1.1). It follows from Lemma 2.1 and $R_{(A, B)^{*}}=L_{(A, B)}, R_{A^{*}}=L_{A}, R_{B^{*}}=L_{B}$ that

$$
\begin{aligned}
X_{1} & =\tilde{X}_{0}+S_{1} L_{G} U L_{G} S_{1}^{*}+L_{A} V_{1}+V_{2} L_{A}, \\
Y_{1} & =\tilde{Y}_{0}-S_{2} L_{G} U L_{G} S_{2}^{*}+L_{B} W_{1}+W_{2} L_{B},
\end{aligned}
$$

where $\tilde{X}_{0}$ and $\tilde{Y}_{0}$ are a special pair solution of (1.1), and $U, V_{1}, V_{2}, W_{1}$, and $W_{2}$ are arbitrary matrices with suitable sizes. Since $\left(\frac{X_{1}+X_{1}^{*}}{2}, \frac{Y_{1}+Y_{1}^{*}}{2}\right)$ is also a pair Hermitian solution of (1.1), we get that

$$
\begin{aligned}
X_{1}= & \frac{1}{2}\left(X_{1}+X_{1}^{*}\right)=\frac{1}{2}\left(\tilde{X}_{0}+\tilde{X}_{0}^{*}\right)+\frac{1}{2} S_{1} L_{G}\left(U+U^{*}\right) L_{G} S_{1}^{*}+\frac{1}{2} L_{A}\left(V_{1}+V_{2}^{*}\right) \\
& +\frac{1}{2}\left(V_{1}+V_{2}^{*}\right)^{*} L_{A}, \\
Y_{1}= & \frac{1}{2}\left(Y_{1}+Y_{1}^{*}\right)=\frac{1}{2}\left(\tilde{Y}_{0}+\tilde{Y}_{0}^{*}\right)-\frac{1}{2} S_{2} L_{G}\left(U+U^{*}\right) L_{G} S_{2}^{*}+\frac{1}{2} L_{B}\left(W_{1}+W_{2}^{*}\right) \\
& +\frac{1}{2}\left(W_{1}+W_{2}^{*}\right)^{*} L_{B} .
\end{aligned}
$$

Putting

$$
\begin{aligned}
X_{0} & =\frac{1}{2}\left(\tilde{X}_{0}+\tilde{X}_{0}^{*}\right), Y_{0}=\frac{1}{2}\left(\tilde{Y}_{0}+\tilde{Y}_{0}^{*}\right), V=\frac{1}{2}\left(V_{1}+V_{2}^{*}\right), \\
W & =\frac{1}{2}\left(W_{1}+W_{2}^{*}\right), Z=\frac{1}{2}\left(U+U^{*}\right),
\end{aligned}
$$

and noting that $X_{0}$ and $Y_{0}$ are a special pair Hermitian solution of (1.1), $Z$ is an arbitrary Hermitian matrix, we get that any pair Hermitian solution $\left(X_{1}, Y_{1}\right)$ of $(1.1)$ has the form of (2.2).

Conversely, it can be verified that a pair of matrices having the form of (2.2) are a pair Hermitian solution of (1.1). Therefore, (2.2) is an expression of the general Hermitian solution to (1.1). 
Tian and Liu in [21] and [12] gave the following extremal ranks of matrix expressions $A-B X B^{*}$ and $A-B X-X^{*} B^{*}$ over a field. The results can be generalized to $\mathbb{H}$.

Lemma 2.3. Let $A \in \mathbb{H}^{m \times m}, B \in \mathbb{H}^{m \times n}$.

1. If $A=A^{*}$, then

$$
\begin{aligned}
& \max _{X=X^{*} \in \mathbb{H}^{n \times n}} r\left(A-B X B^{*}\right)=r[A, B], \\
& \min _{X=X^{*} \in \mathbb{H}^{n \times n}} r\left(A-B X B^{*}\right)=2 r[A, B]-r\left[\begin{array}{cc}
A & B \\
B^{*} & 0
\end{array}\right] ; \\
& \max _{X \in \mathbb{H}^{n \times m}} r\left(A+B X+X^{*} B^{*}\right)=\min \left\{m, r\left[\begin{array}{cc}
A & B \\
B^{*} & 0
\end{array}\right]\right\}, \\
& \min _{X \in \mathbb{H}^{n \times m}} r\left(A+B X+X^{*} B^{*}\right)=r\left[\begin{array}{cc}
A & B \\
B^{*} & 0
\end{array}\right]-2 r(B) .
\end{aligned}
$$

2. If $A=-A^{*}$, then

$$
\begin{aligned}
\max _{X=-X^{*} \in \mathbb{H}^{n \times n}} r\left(A-B X B^{*}\right) & =r[A, B], \\
\min _{X=-X^{*} \in \mathbb{H}^{n \times n}} r\left(A-B X B^{*}\right) & =2 r[A, B]-r\left[\begin{array}{cc}
A & B \\
-B^{*} & 0
\end{array}\right] ; \\
\max _{X \in \mathbb{H}^{n \times m}} r\left(A+B X-X^{*} B^{*}\right) & =\min \left\{m, r\left[\begin{array}{cc}
A & B \\
-B^{*} & 0
\end{array}\right]\right\}, \\
\min _{X \in \mathbb{H}^{n \times m}} r\left(A+B X-X^{*} B^{*}\right) & =r\left[\begin{array}{cc}
A & B \\
-B^{*} & 0
\end{array}\right]-2 r(B) .
\end{aligned}
$$

The following lemma is due to Marsaglia and Styan [14], which can be generalized to $\mathbb{H}$.

Lemma 2.4. Let $A \in \mathbb{H}^{m \times n}, B \in \mathbb{H}^{m \times k}, C \in \mathbb{H}^{l \times n}, D \in \mathbb{H}^{j \times k}$ and $E \in \mathbb{H}^{l \times i}$. Then they satisfy the following rank equalities:

(a) $r\left(C L_{A}\right)=r\left[\begin{array}{l}A \\ C\end{array}\right]-r(A)$,

(b) $r\left[\begin{array}{ll}B & A L_{C}\end{array}\right]=r\left[\begin{array}{cc}B & A \\ 0 & C\end{array}\right]-r(C)$,

(c) $r\left[\begin{array}{c}C \\ R_{B} A\end{array}\right]=r\left[\begin{array}{ll}C & 0 \\ A & B\end{array}\right]-r(B)$.

Now we give one of the main theorems in this paper.

TheOrem 2.5. Suppose that the matrix equation (1.1), where $A \in \mathbb{H}^{m \times n}, B \in$ $\mathbb{H}^{m \times p}, C \in \mathbb{H}^{m \times m}, C=C^{*}, X \in \mathbb{H}^{n \times n}$, and $Y \in \mathbb{H}^{p \times p}$, has a Hermitian solution.

(a) The maximal and minimal ranks of the general Hermitian solution to (1.1) 
are given by:

$$
\begin{aligned}
& \max _{\substack{A X A^{*}+B Y B^{*}=C \\
X=X^{*}}} r(X)=\min \{n, r[B, C]+2 n-r(A)-r(G)\}, \\
& \min _{\substack{A X A^{*}+B Y B^{*} B^{*}=C \\
X^{*}}} r(X)=2 r[B, C]-r\left[\begin{array}{cc}
C & B \\
B^{*} & 0
\end{array}\right] ; \\
& \max _{\substack{A X A^{*}+B Y B^{*}=C \\
Y=Y^{*}}} r(Y)=\min \{p, r[A, C]+2 p-r(B)-r(G)\}, \\
& \min _{\substack{A X A^{*}+B Y^{*} B^{*}=C \\
Y=Y^{*}}} r(Y)=2 r[A, C]-r\left[\begin{array}{cc}
C & A \\
A^{*} & 0
\end{array}\right] .
\end{aligned}
$$

(b) The rank of the general Hermitian solution $X$ to (1.1) is invariant if and only if

$$
2 r[B, C]-r\left[\begin{array}{cc}
C & B \\
B^{*} & 0
\end{array}\right]=n
$$

or

$$
r[B, C]+r(A)+r(G)=r\left[\begin{array}{cc}
C & B \\
B^{*} & 0
\end{array}\right]+2 n .
$$

The rank of the general Hermitian solution $Y$ to (1.1) is invariant if and only if

$$
2 r[A, C]-r\left[\begin{array}{cc}
C & A \\
A^{*} & 0
\end{array}\right]=p
$$

or

$$
r[A, C]+r(B)+r(G)=r\left[\begin{array}{cc}
C & A \\
A^{*} & 0
\end{array}\right]+2 p .
$$

Proof. (a) Applying Lemma 2.2 and Lemma 2.3 to $X$ of (2.2), we get that

$$
\begin{aligned}
\max _{A X A^{*}+B Y B^{*}=C} r(X) & =\max _{Z=Z^{*}, V} r\left(X_{0}+S_{1} L_{G} Z L_{G} S_{1}^{*}+L_{A} V+V^{*} L_{A}\right) \\
& =\min \left\{n, \max _{Z=Z^{*}} r\left[\begin{array}{cc}
X_{0}+S_{1} L_{G} Z L_{G} S_{1}^{*} & L_{A} \\
L_{A} & 0
\end{array}\right]\right\} \\
& =\min \left\{n, \max _{Z=Z^{*}} r\left(\left[\begin{array}{cc}
X_{0} & L_{A} \\
L_{A} & 0
\end{array}\right]+\left[\begin{array}{c}
S_{1} L_{G} \\
0
\end{array}\right] Z\left[\begin{array}{ll}
L_{G} S_{1}^{*} & 0
\end{array}\right]\right)\right\} \\
(2.8) \quad & \min \left\{n, r\left[\begin{array}{ccc}
X_{0} & L_{A} & S_{1} L_{G} \\
L_{A} & 0 & 0
\end{array}\right]\right\}
\end{aligned}
$$




$$
\begin{aligned}
\min _{\substack{A X A^{*}+B Y X^{*} \\
X=X^{*}}} r(X) & =\min _{Z=Z^{*}, V} r\left(X_{0}+S_{1} L_{G} Z L_{G} S_{1}^{*}+L_{A} V+V^{*} L_{A}\right) \\
& =\min _{Z=Z^{*}} r\left[\begin{array}{cc}
X_{0}+S_{1} L_{G} Z L_{G} S_{1}^{*} & L_{A} \\
& L_{A} \\
0
\end{array}\right]-2 r\left(L_{A}\right) \\
& =\min _{Z=Z^{*}} r\left(\left[\begin{array}{cc}
X_{0} & L_{A} \\
L_{A} & 0
\end{array}\right]+\left[\begin{array}{c}
S_{1} L_{G} \\
0
\end{array}\right] Z\left[\begin{array}{ccc}
L_{G} S_{1}^{*} & 0
\end{array}\right]\right)-2 r\left(L_{A}\right) \\
& =2 r\left[\begin{array}{ccc}
X_{0} & L_{A} & S_{1} L_{G} \\
L_{A} & 0 & 0
\end{array}\right]-r\left[\begin{array}{ccc}
X_{0} & L_{A} & S_{1} L_{G} \\
L_{A} & 0 & 0 \\
L_{G} S_{1}^{*} & 0 & 0
\end{array}\right] \\
& -2 r\left(L_{A}\right) .
\end{aligned}
$$

By Lemma 2.4, block Gaussian elimination, and $A X_{0} A^{*}+B Y_{0} B^{*}=C$, we have that $r\left(L_{A}\right)=n-r(A)$,

$$
\begin{aligned}
r\left[\begin{array}{ccc}
X_{0} & S_{1} L_{G} & L_{A} \\
L_{A} & 0 & 0
\end{array}\right]=r\left[\begin{array}{cccc}
X_{0} & I_{n} & S_{1} & 0 \\
I_{n} & 0 & 0 & A^{*} \\
0 & A & 0 & 0 \\
0 & 0 & G & 0
\end{array}\right]-r(A)-r\left(A^{*}\right)-r(G) \\
=r[B, C]+2 n-r(A)-r(G), \\
r\left[\begin{array}{ccc}
X_{0} & L_{A} & S_{1} L_{G} \\
L_{A} & 0 & 0 \\
L_{G} S_{1}^{*} & 0 & 0
\end{array}\right]=r\left[\begin{array}{ccccc}
X_{0} & I_{n} & S_{1} & 0 & 0 \\
I_{n} & 0 & 0 & A^{*} & 0 \\
S_{1}^{*} & 0 & 0 & 0 & G^{*} \\
0 & A & 0 & 0 & 0 \\
0 & 0 & G & 0 & 0
\end{array}\right]-r(A)-r\left(A^{*}\right)-2 r(G) \\
=r\left[\begin{array}{cc}
C & B \\
B^{*} & 0
\end{array}\right]+2 n-2 r(G) .
\end{aligned}
$$

Substituting above two equalities into (2.8) and (2.9) yields (2.4) and (2.5), respectively.

Similarly, we can get the corresponding results on $Y$.

(b) The ranks of $X, Y$, expressed as (2.2), in the general pair Hermitian solution to (1.1) are invariant if and only if

$$
\max r(X)-\min r(X)=0, \max r(Y)-\min r(Y)=0 .
$$

Hence result (b) follows from (2.4)-(2.7), and (2.10).

Similarly, we can get the following.

Theorem 2.6. Let $A \in \mathbb{H}^{m \times n}, B \in \mathbb{H}^{m \times p}, C \in \mathbb{H}^{m \times m}, C=-C^{*}, X \in \mathbb{H}^{n \times n}$, and $Y \in \mathbb{H}^{p \times p}$. Suppose that (1.1) has a skew-Hermitian solution over $\mathbb{H}$. 
(a) The maximal and minimal ranks of the general skew-Hermitian solution to (1.1) are given by

$$
\begin{aligned}
& \max _{A X A^{*}+B Y B^{*}=C} r(X)=\min \{n, r[B, C]+2 n-r(A)-r(G)\},
\end{aligned}
$$

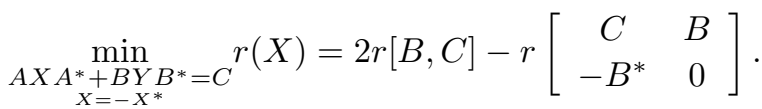

$$
\begin{aligned}
& \max _{\substack{A X A^{*}+B Y Y^{*}=C \\
Y=-Y^{*}}} r(Y)=\min \{p, r[A, C]+2 p-r(B)-r(G)\} ;
\end{aligned}
$$

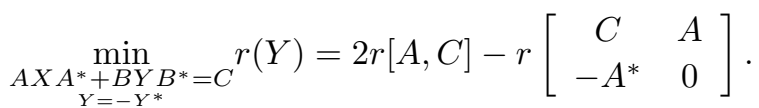

(b) The rank of the general skew-Hermitian solution $X$ to (1.1) is invariant if and only if

$$
\begin{gathered}
2 r[B, C]-r\left[\begin{array}{cc}
C & B \\
-B^{*} & 0
\end{array}\right]=n, \\
\text { or } r[B, C]+r(A)+r(G)=r\left[\begin{array}{cc}
C & B \\
-B^{*} & 0
\end{array}\right]+2 n .
\end{gathered}
$$

The rank of the general skew-Hermitian solution $Y$ to (1.1) is invariant if and only if

$$
2 r[A, C]-r\left[\begin{array}{cc}
C & A \\
-A^{*} & 0
\end{array}\right]=p \text {, or } r[A, C]+r(B)+r(G)=r\left[\begin{array}{cc}
C & A \\
-A^{*} & 0
\end{array}\right]+2 p .
$$

3. Extreme ranks of the real matrices in a Hermitian solution to (1.1) over $\mathbb{H}$. In this section, we consider the maximal and minimal ranks of real matrices $X_{i}, Y_{i}$ in a pair Hermitian solution $X=X_{1}+X_{2} i+X_{3} j+X_{4} k \in \mathbb{H}^{n \times n}$ and $Y=$ $Y_{1}+Y_{2} i+Y_{3} j+Y_{4} k \in \mathbb{H}^{p \times p}$ to (1.1) where

$A=A_{1}+A_{2} i+A_{3} j+A_{4} k, B=B_{1}+B_{2} i+B_{3} j+B_{4} k, C=C_{1}+C_{2} i+C_{3} j+C_{4} k$, $A_{i}, B_{i}, C_{i}, i=1, \cdots, 4$, are real matrices with suitable sizes.

For an arbitrary quaternion matrix $M=M_{1}+M_{2} i+M_{3} j+M_{4} k$, we now define a map $\phi(\cdot)$, from $\mathbb{H}^{m \times n}$ to $\mathbb{R}^{4 m \times 4 n}$, by

$$
\phi(M)=\left[\begin{array}{cccc}
M_{1} & -M_{2} & -M_{3} & -M_{4} \\
M_{2} & M_{1} & -M_{4} & M_{3} \\
M_{3} & M_{4} & M_{1} & -M_{2} \\
M_{4} & -M_{3} & M_{2} & M_{1}
\end{array}\right] .
$$

By (3.1), it is easy to verify that $\phi(\cdot)$ satisfies the following properties: 
(a) $M=N \Longleftrightarrow \phi(M)=\phi(N)$.

(b) $\phi(M+N)=\phi(M)+\phi(N), \phi(M N)=\phi(M) \phi(N), \phi(k M)=k \phi(M), k \in \mathbb{R}$.

(c) $\phi\left(M^{*}\right)=\phi^{T}(M), \phi\left(M^{\dagger}\right)=\phi^{\dagger}(M)$.

(d) $\phi(M)=T_{m}^{-1} \phi(M) T_{n}=R_{m}^{-1} \phi(M) R_{n}=S_{m}^{-1} \phi(M) S_{n}$, where for $t=m, n$,

$$
\begin{gathered}
R_{t}=\left[\begin{array}{cc}
0 & -I_{2 t} \\
I_{2 t} & 0
\end{array}\right], S_{t}=\left[\begin{array}{cccc}
0 & 0 & 0 & -I_{t} \\
0 & 0 & I_{t} & 0 \\
0 & -I_{t} & 0 & 0 \\
I_{t} & 0 & 0 & 0
\end{array}\right], \\
T_{t}=\left[\begin{array}{cccc}
0 & -I_{t} & 0 & 0 \\
I_{t} & 0 & 0 & 0 \\
0 & 0 & 0 & I_{t} \\
0 & 0 & -I_{t} & 0
\end{array}\right] .
\end{gathered}
$$

(e) $r[\phi(M)]=4 r(M)$.

(f) $M^{*}=M \Leftrightarrow \phi^{T}(M)=\phi(M)$, and $M^{*}=-M \Leftrightarrow \phi^{T}(M)=-\phi(M)$.

In the following theorems and corollaries, $X_{0}, Y_{0}, S_{1}, S_{2}$, and $G$ are defined as in Lemma 2.2.

THEOREM 3.1. The matrix equation (1.1) has a Hermitian solution over $\mathbb{H}$ if and only if the matrix equation

$$
\phi(A)\left(X_{i j}\right)_{4 \times 4} \phi^{T}(A)+\phi(B)\left(Y_{i j}\right)_{4 \times 4} \phi^{T}(B)=\phi(C), i, j=1,2,3,4,
$$

has a symmetric solution over $\mathbb{R}$. In this case, the general Hermitian solution of (1.1) over $\mathbb{H}$ can be written as:

$$
\begin{aligned}
X & =X_{1}+X_{2} i+X_{3} j+X_{4} k \\
& =\frac{1}{4}\left(X_{11}+X_{22}+X_{33}+X_{44}\right)+\frac{1}{4}\left(X_{12}^{T}-X_{12}+X_{34}^{T}-X_{34}\right) i \\
& +\frac{1}{4}\left(X_{13}^{T}-X_{13}+X_{24}-X_{24}^{T}\right) j+\frac{1}{4}\left(X_{14}^{T}-X_{14}+X_{23}^{T}-X_{23}\right) k, \\
Y & =Y_{1}+Y_{2} i+Y_{3} j+Y_{4} k \\
& =\frac{1}{4}\left(Y_{11}+Y_{22}+Y_{33}+Y_{44}\right)+\frac{1}{4}\left(Y_{12}^{T}-Y_{12}+Y_{34}^{T}-Y_{34}\right) i \\
& +\frac{1}{4}\left(Y_{13}^{T}-Y_{13}+Y_{24}-Y_{24}^{T}\right) j+\frac{1}{4}\left(Y_{14}^{T}-Y_{14}+Y_{23}^{T}-Y_{23}\right) k,
\end{aligned}
$$

where $X_{t t}=X_{t t}^{T}, Y_{t t}=Y_{t t}^{T}, t=1,2,3,4 ; X_{1 j}^{T}=X_{j 1}, Y_{1 j}^{T}=Y_{j 1}, j=2,3,4 ; X_{2 j}^{T}=$ $X_{j 2}, Y_{2 j}^{T}=Y_{j 2}, j=3,4 ; X_{34}^{T}=X_{43}, Y_{34}^{T}=Y_{43}$ are the general solutions of (3.2) over 
$\mathbb{R}$. Written in explicit forms, $X_{i}, Y_{i}, i=1,2,3$, 4 , in (3.3) and (3.4) are

$$
\begin{aligned}
X_{1} & =\frac{1}{4} P_{1} \phi\left(X_{0}\right) P_{1}^{T}+\frac{1}{4} P_{2} \phi\left(X_{0}\right) P_{2}^{T}+\frac{1}{4} P_{3} \phi\left(X_{0}\right) P_{3}^{T}+\frac{1}{4} P_{4} \phi\left(X_{0}\right) P_{4}^{T} \\
& +\frac{1}{4}\left[P_{1}, P_{2}, P_{3}, P_{4}\right] E_{1} Z E_{1}^{T}\left[P_{1}, P_{2}, P_{3}, P_{4}\right]^{T} \\
& +\left[P_{1}, P_{2}, P_{3}, P_{4}\right] L_{\phi(A)}\left[\begin{array}{c}
V_{1} \\
V_{2} \\
V_{3} \\
V_{4}
\end{array}\right]+\left[\begin{array}{c}
V_{1} \\
V_{2} \\
V_{3} \\
V_{4}
\end{array}\right] L_{\phi(A)}^{T}\left[P_{1}, P_{2}, P_{3}, P_{4}\right]^{T},
\end{aligned}
$$

$$
\begin{aligned}
X_{2} & =\frac{1}{4} P_{2} \phi\left(X_{0}\right) P_{1}^{T}-\frac{1}{4} P_{1} \phi\left(X_{0}\right) P_{2}^{T}+\frac{1}{4} P_{4} \phi\left(X_{0}\right) P_{3}^{T}-\frac{1}{4} P_{3} \phi\left(X_{0}\right) P_{4}^{T} \\
& -\frac{1}{4}\left[P_{1},-P_{2}, P_{3},-P_{4}\right] E_{1} Z E_{1}^{T}\left[P_{2}, P_{1}, P_{4}, P_{3}\right]^{T} \\
& -\left[-P_{2}, P_{1},-P_{4}, P_{3}\right] L_{\phi(A)}\left[\begin{array}{c}
V_{1} \\
V_{2} \\
V_{3} \\
V_{4}
\end{array}\right]+\left[\begin{array}{c}
V_{1} \\
V_{2} \\
V_{3} \\
V_{4}
\end{array}\right]^{T} L_{\phi(A)}^{T}\left[-P_{2}, P_{1},-P_{4}, P_{3}\right]^{T},
\end{aligned}
$$

$$
\begin{aligned}
& X_{3}=\frac{1}{4} P_{3} \phi\left(X_{0}\right) P_{1}^{T}-\frac{1}{4} P_{1} \phi\left(X_{0}\right) P_{3}^{T}+\frac{1}{4} P_{2} \phi\left(X_{0}\right) P_{4}^{T}-\frac{1}{4} P_{4} \phi\left(X_{0}\right) P_{2}^{T} \\
& -\frac{1}{4}\left[P_{1},-P_{2},-P_{3}, P_{4}\right] E_{1} Z E_{1}^{T}\left[P_{3}, P_{4}, P_{1}, P_{2}\right]^{T} \\
& -\left[-P_{3}, P_{1},-P_{2}, P_{4}\right] L_{\phi(A)}\left[\begin{array}{c}
V_{1} \\
V_{2} \\
V_{3} \\
V_{4}
\end{array}\right]+\left[\begin{array}{c}
V_{1} \\
V_{2} \\
V_{3} \\
V_{4}
\end{array}\right]^{T} L_{\phi(A)}^{T}\left[-P_{3}, P_{1},-P_{2}, P_{4}\right]^{T}, \\
& X_{4}=\frac{1}{4} P_{4} \phi\left(X_{0}\right) P_{1}^{T}-\frac{1}{4} P_{1} \phi\left(X_{0}\right) P_{4}^{T}+\frac{1}{4} P_{3} \phi\left(X_{0}\right) P_{2}^{T}-\frac{1}{4} P_{2} \phi\left(X_{0}\right) P_{3}^{T} \\
& -\frac{1}{4}\left[-P_{1},-P_{2}, P_{3}, P_{4}\right] E_{1} Z E_{1}^{T}\left[P_{4}, P_{3}, P_{2}, P_{1}\right]^{T} \\
& -\left[-P_{4}, P_{1},-P_{3}, P_{2}\right] L_{\phi(A)}\left[\begin{array}{c}
V_{1} \\
V_{2} \\
V_{3} \\
V_{4}
\end{array}\right]+\left[\begin{array}{c}
V_{1} \\
V_{2} \\
V_{3} \\
V_{4}
\end{array}\right]^{T} L_{\phi(A)}^{T}\left[-P_{4}, P_{1},-P_{3}, P_{2}\right]^{T},
\end{aligned}
$$




$$
\begin{aligned}
Y_{1} & =\frac{1}{4} Q_{1} \phi\left(Y_{0}\right) Q_{1}^{T}+\frac{1}{4} Q_{2} \phi\left(Y_{0}\right) Q_{2}^{T}+\frac{1}{4} Q_{3} \phi\left(Y_{0}\right) Q_{3}^{T}+\frac{1}{4} Q_{4} \phi\left(Y_{0}\right) Q_{4}^{T} \\
& -\frac{1}{4}\left[Q_{1}, Q_{2}, Q_{3}, Q_{4}\right] E_{2} Z E_{2}^{T}\left[Q_{1}, Q_{2}, Q_{3}, Q_{4}\right]^{T} \\
& +\left[Q_{1}, Q_{2}, Q_{3}, Q_{4}\right] L_{\phi(B)}\left[\begin{array}{c}
W_{1} \\
W_{2} \\
W_{3} \\
W_{4}
\end{array}\right]+\left[\begin{array}{c}
W_{1} \\
W_{2} \\
W_{3} \\
W_{4}
\end{array}\right] L_{\phi(B)}^{T}\left[Q_{1}, Q_{2}, Q_{3}, Q_{4}\right]^{T},
\end{aligned}
$$

$$
\begin{aligned}
Y_{2} & =\frac{1}{4} Q_{2} \phi\left(Y_{0}\right) Q_{1}^{T}-\frac{1}{4} Q_{1} \phi\left(Y_{0}\right) Q_{2}^{T}+\frac{1}{4} Q_{4} \phi\left(Y_{0}\right) Q_{3}^{T}-\frac{1}{4} Q_{3} \phi\left(Y_{0}\right) Q_{4}^{T} \\
& +\frac{1}{4}\left[Q_{1},-Q_{2}, Q_{3},-Q_{4}\right] E_{2} Z E_{2}^{T}\left[Q_{2}, Q_{1}, Q_{4}, Q_{3}\right]^{T}
\end{aligned}
$$

$$
-\left[-Q_{2}, Q_{1},-Q_{4}, Q_{3}\right] L_{\phi(B)}\left[\begin{array}{c}
W_{1} \\
W_{2} \\
W_{3} \\
W_{4}
\end{array}\right]+\left[\begin{array}{c}
W_{1} \\
W_{2} \\
W_{3} \\
W_{4}
\end{array}\right]^{T} L_{\phi(B)}^{T}\left[-Q_{2}, Q_{1},-Q_{4}, Q_{3}\right]^{T},
$$

$$
\begin{aligned}
Y_{3} & =\frac{1}{4} Q_{3} \phi\left(Y_{0}\right) Q_{1}^{T}-\frac{1}{4} Q_{1} \phi\left(Y_{0}\right) Q_{3}^{T}+\frac{1}{4} Q_{2} \phi\left(Y_{0}\right) Q_{4}^{T}-\frac{1}{4} Q_{4} \phi\left(Y_{0}\right) Q_{2}^{T} \\
& +\frac{1}{4}\left[Q_{1},-Q_{2},-Q_{3}, Q_{4}\right] E_{2} Z E_{2}^{T}\left[Q_{3}, Q_{4}, Q_{1}, Q_{2}\right]^{T}
\end{aligned}
$$

$$
-\left[-Q_{3}, Q_{1},-Q_{2}, Q_{4}\right] L_{\phi(B)}\left[\begin{array}{l}
W_{1} \\
W_{2} \\
W_{3} \\
W_{4}
\end{array}\right]+\left[\begin{array}{c}
W_{1} \\
W_{2} \\
W_{3} \\
W_{4}
\end{array}\right]^{T} L_{\phi(B)}^{T}\left[-Q_{3}, Q_{1},-Q_{2}, Q_{4}\right]^{T},
$$

$$
\begin{aligned}
Y_{4} & =\frac{1}{4} Q_{4} \phi\left(Y_{0}\right) Q_{1}^{T}-\frac{1}{4} Q_{1} \phi\left(Y_{0}\right) Q_{4}^{T}+\frac{1}{4} Q_{3} \phi\left(Y_{0}\right) Q_{2}^{T}-\frac{1}{4} Q_{2} \phi\left(Y_{0}\right) Q_{3}^{T} \\
& +\frac{1}{4}\left[-Q_{1},-Q_{2}, Q_{3}, Q_{4}\right] E_{2} Z E_{2}^{T}\left[Q_{4}, Q_{3}, Q_{2}, Q_{1}\right]^{T}
\end{aligned}
$$

$$
-\left[-Q_{4}, Q_{1},-Q_{3}, Q_{2}\right] L_{\phi(B)}\left[\begin{array}{c}
W_{1} \\
W_{2} \\
W_{3} \\
W_{4}
\end{array}\right]+\left[\begin{array}{c}
W_{1} \\
W_{2} \\
W_{3} \\
W_{4}
\end{array}\right]^{T} L_{\phi(B)}^{T}\left[-Q_{4}, Q_{1},-Q_{3}, Q_{2}\right]^{T},
$$


where

$$
\begin{aligned}
P_{1} & =\left[I_{n}, 0,0,0\right], P_{2}=\left[0, I_{n}, 0,0\right], P_{3}=\left[0,0, I_{n}, 0\right], P_{4}=\left[0,0,0, I_{n}\right], \\
Q_{1} & =\left[I_{p}, 0,0,0\right], Q_{2}=\left[0, I_{p}, 0,0\right], Q_{3}=\left[0,0, I_{p}, 0\right], Q_{4}=\left[0,0,0, I_{p}\right], \\
E_{1} & =\phi\left(S_{1}\right) L_{\phi(G)}, E_{2}=\phi\left(S_{2}\right) L_{\phi(G)} ;
\end{aligned}
$$

$Z$ is arbitrary real symmetric matrix, and $V_{1}, V_{2}, V_{3}, V_{4}, W_{1}, W_{2}, W_{3}$, and $W_{4}$ are arbitrary real matrices with compatible sizes.

Proof. Suppose that (1.1) has a Hermitian solution

$$
X=X_{1}+X_{2} i+X_{3} j+X_{4} k, Y=Y_{1}+Y_{2} i+Y_{3} j+Y_{4} k
$$

over $\mathbb{H}$. Applying properties $(a)$ and $(b)$ of $\phi(\cdot)$ to $(1.1)$ yields

$$
\phi(A) \phi(X) \phi^{T}(A)+\phi(B) \phi(Y) \phi^{T}(B)=\phi(C), \phi^{T}(X)=\phi(X), \phi^{T}(Y)=\phi(Y),
$$

which implies that $\phi(X), \phi(Y)$ are real symmetric solutions to (3.2). Conversely, suppose that (3.2) has a real symmetric solution

$$
X=\left[\begin{array}{llll}
X_{11} & X_{12} & X_{13} & X_{14} \\
X_{21} & X_{22} & X_{23} & X_{24} \\
X_{31} & X_{32} & X_{33} & X_{24} \\
X_{41} & X_{42} & X_{43} & X_{44}
\end{array}\right], Y=\left[\begin{array}{llll}
Y_{11} & Y_{12} & Y_{13} & Y_{14} \\
Y_{21} & Y_{22} & Y_{23} & Y_{24} \\
Y_{31} & Y_{32} & Y_{33} & Y_{24} \\
Y_{41} & Y_{42} & Y_{43} & Y_{44}
\end{array}\right],
$$

i.e.

$$
\phi(A) X \phi^{T}(A)+\phi(B) Y \phi^{T}(B)=\phi(C), X^{T}=X, Y^{T}=Y .
$$

By property $(d)$ of $\phi(\cdot)$, we have that

$$
\begin{aligned}
T_{m}^{-1} \phi(A) T_{n} X T_{n}^{-1} \phi^{T}(A) T_{m}+T_{m}^{-1} \phi(B) T_{p} Y T_{p}^{-1} \phi^{T}(B) T_{m} & =T_{m}^{-1} \phi(C) T_{m}, \\
R_{m}^{-1} \phi(A) R_{n} X R_{n}^{-1} \phi^{T}(A) R_{m}+R_{m}^{-1} \phi(B) R_{p} Y R_{p}^{-1} \phi^{T}(B) R_{m} & =R_{m}^{-1} \phi(C) R_{m}, \\
S_{m}^{-1} \phi(A) S_{n} X S_{n}^{-1} \phi^{T}(A) S_{m}+S_{m}^{-1} \phi(B) S_{p} Y S_{p}^{-1} \phi^{T}(B) S_{m} & =S_{m}^{-1} \phi(C) S_{m} .
\end{aligned}
$$

Hence

$$
\begin{aligned}
\phi(A) T_{n} X T_{n}^{-1} \phi^{T}(A)+\phi(B) T_{p} Y T_{p}^{-1} \phi^{T}(B) & =\phi(C), \\
\phi(A) R_{n} X R_{n}^{-1} \phi^{T}(A)+\phi(B) R_{p} Y R_{p}^{-1} \phi^{T}(B) & =\phi(C), \\
\phi(A) S_{n} X S_{n}^{-1} \phi^{T}(A)+\phi(B) S_{p} Y S_{p}^{-1} \phi^{T}(B) & =\phi(C),
\end{aligned}
$$

implying $T_{n} X T_{n}^{-1}, T_{p} Y T_{p}^{-1}, R_{n} X R_{n}^{-1}, R_{p} Y R_{p}^{-1}, S_{n} X S_{n}^{-1}$, and $S_{p} Y S_{p}^{-1}$ are also symmetric solutions of (3.2) over $\mathbb{R}$. Thus,

$$
\frac{1}{4}\left(X+T_{n} X T_{n}^{-1}+R_{n} X R_{n}^{-1}+S_{n} X S_{n}^{-1}\right), \frac{1}{4}\left(Y+T_{p} Y T_{p}^{-1}+R_{p} Y R_{p}^{-1}+S_{p} Y S_{p}^{-1}\right)
$$


are symmetric solutions of $(3.2)$, where

$$
X+T_{p} X T_{p}^{-1}+R_{p} X R_{p}^{-1}+S_{p} X S_{p}^{-1}=\left(\widetilde{X_{i j}}\right)_{4 \times 4}, i, j=1,2,3,4
$$

and

$$
\begin{aligned}
& \widetilde{X_{11}}=X_{11}+X_{22}+X_{33}+X_{44}, \widetilde{X_{12}}=X_{12}-X_{12}^{T}+X_{34}-X_{34}^{T}, \\
& \widetilde{X_{13}}=X_{13}-X_{13}^{T}+X_{24}^{T}-X_{24}, \widetilde{X_{14}}=X_{14}-X_{14}^{T}+X_{23}-X_{23}^{T}, \\
& \widetilde{X_{21}}=X_{12}^{T}-X_{12}+X_{34}^{T}-X_{34}, \widetilde{X_{22}}=X_{11}+X_{22}+X_{33}+X_{44}, \\
& \widetilde{X_{23}}=X_{14}-X_{14}^{T}+X_{23}-X_{23}^{T}, \widetilde{X_{24}}=X_{13}^{T}-X_{13}+X_{24}-X_{24}^{T}, \\
& \widetilde{X_{31}}=X_{13}^{T}-X_{13}+X_{24}-X_{24}^{T}, \widetilde{X_{32}}=X_{14}^{T}-X_{14}+X_{23}^{T}-X_{23}, \\
& \widetilde{X_{33}}=X_{11}+X_{22}+X_{33}+X_{44}, \widetilde{X_{34}}=X_{12}-X_{12}^{T}+X_{34}-X_{34}^{T}, \\
& \widetilde{X_{41}}=X_{14}^{T}-X_{14}+X_{23}^{T}-X_{23}, \widetilde{X_{42}}=X_{13}-X_{13}^{T}+X_{24}^{T}-X_{24}, \\
& \widetilde{X_{43}}=X_{12}^{T}-X_{12}+X_{34}^{T}-X_{34}, \widetilde{X_{44}}=X_{11}+X_{22}+X_{33}+X_{44} .
\end{aligned}
$$

$Y+T_{p} Y T_{p}^{-1}+R_{p} Y R_{p}^{-1}+S_{p} Y S_{p}^{-1}$ has a form similar to $\left(\widetilde{X_{i j}}\right)_{4 \times 4}$. We omit it here for simplicity.

Let

$$
\begin{aligned}
\hat{X}= & \frac{1}{4}\left(X_{11}+X_{22}+X_{33}+X_{44}\right)+\frac{1}{4}\left(X_{12}^{T}-X_{12}+X_{34}^{T}-X_{34}\right) i \\
+ & \frac{1}{4}\left(X_{13}^{T}-X_{13}+X_{24}-X_{24}^{T}\right) j+\frac{1}{4}\left(X_{14}^{T}-X_{14}+X_{23}^{T}-X_{23}\right) k, \\
\hat{Y} & =\frac{1}{4}\left(Y_{11}+Y_{22}+Y_{33}+Y_{44}\right)+\frac{1}{4}\left(Y_{12}^{T}-Y_{12}+Y_{34}^{T}-Y_{34}\right) i \\
& +\frac{1}{4}\left(Y_{13}^{T}-Y_{13}+Y_{24}-Y_{24}^{T}\right) j+\frac{1}{4}\left(Y_{14}^{T}-Y_{14}+Y_{23}^{T}-Y_{23}\right) k .
\end{aligned}
$$

Then by (3.1),

$$
\begin{aligned}
\phi(\hat{X}) & =\frac{1}{4}\left(X+T_{n} X T_{n}^{-1}+R_{n} X R_{n}^{-1}+S_{n} X S_{n}^{-1}\right), \\
\phi(\hat{Y}) & =\frac{1}{4}\left(Y+T_{p} Y T_{p}^{-1}+R_{p} Y R_{p}^{-1}+S_{p} Y S_{p}^{-1}\right),
\end{aligned}
$$

we have that $\hat{X}, \hat{Y}$ are a pair Hermitian solution of (1.1) by the property $(a)$. Observe that $X_{i j}$ and $Y_{i j}, i, j=1,2,3,4$ in $(3.2)$ can be written as

$$
X_{i j}=P_{i} X P_{j}^{T}, Y_{i j}=Q_{i} Y Q_{j}^{T} .
$$

From Lemma 2.2, the general solutions to (3.2) can be written as

$$
\begin{aligned}
X & =\phi\left(X_{0}\right)+\phi\left(S_{1}\right) L_{\phi(G)} Z L_{\phi(G)} \phi^{T}\left(S_{1}\right)+4 L_{\phi(A)}\left[V_{1}, V_{2}, V_{3}, V_{4}\right] \\
& +4\left[V_{1}, V_{2}, V_{3}, V_{4}\right]^{T} L_{\phi(A)}, \\
Y & =\phi\left(Y_{0}\right)-\phi\left(S_{2}\right) L_{\phi(G)} Z L_{\phi(G)} \phi^{T}\left(S_{2}\right)+4 L_{\phi(B)}\left[W_{1}, W_{2}, W_{3}, W_{4}\right] \\
& +4\left[W_{1}, W_{2}, W_{3}, W_{4}\right]^{T} L_{\phi(B)},
\end{aligned}
$$


where $Z$ is an arbitrary real symmetric matrix and $V_{1}, V_{2}, V_{3}, V_{4}, W_{1}, W_{2}, W_{3}$, and $W_{4}$ are arbitrary with suitable sizes. Hence, for $i, j=1,2,3,4$,

$$
\begin{gathered}
X_{i j}=P_{i} \phi\left(X_{0}\right) P_{j}^{T}+P_{i} \phi\left(S_{1}\right) L_{\phi(G)} Z L_{\phi(G)} \phi^{T}\left(S_{1}\right) P_{j}^{T}+4 P_{i} L_{\phi(A)} V_{j}+4 V_{i}^{T} L_{\phi(A)} P_{j}^{T}, \\
Y_{i j}=Q_{i} \phi\left(Y_{0}\right) Q_{j}^{T}-Q_{i} \phi\left(S_{2}\right) L_{\phi(G)} Z L_{\phi(G)} \phi^{T}\left(S_{2}\right) Q_{j}^{T}+4 Q_{i} L_{\phi(B)} W_{j}+4 W_{i}^{T} L_{\phi(B)} Q_{j}^{T} .
\end{gathered}
$$

Substituting them into (3.3) and (3.4) yields real matrices $X_{i}$ and $Y_{i}, i=1,2,3,4$ in (3.5)-(3.12).

Now we consider the maximal and minimal ranks of real matrices $X_{i}, Y_{i}$ in Hermitian solutions $X=X_{1}+X_{2} i+X_{3} j+X_{4} k$ and $Y=Y_{1}+Y_{2} i+Y_{3} j+Y_{4} k$ to (1.1).

THEOREM 3.2. Suppose the matrix equation (1.1) has a Hermitian solution over $\mathbb{H}$, and for $i, j=1,2,3,4$,

$$
\begin{aligned}
& J_{i}=\left\{\begin{array}{l|c}
X_{i} \in \mathbb{R}^{n \times n} & \left.\begin{array}{c}
A\left(X_{1}+X_{2} i+X_{3} j+X_{4} k\right) A^{*} \\
+B\left(Y_{1}+Y_{2} i+Y_{3} j+Y_{4} k\right) B^{*}=C
\end{array}\right\}, \\
T_{j}=\left\{Y_{j} \in \mathbb{R}^{p \times p}\right. & \left.\begin{array}{c}
A\left(X_{1}+X_{2} i+X_{3} j+X_{4} k\right) A^{*} \\
+B\left(Y_{1}+Y_{2} i+Y_{3} j+Y_{4} k\right) B^{*}=C
\end{array}\right\} .
\end{array}\right.
\end{aligned}
$$

Then we have the following:

(a) The maximal and minimal ranks of $X_{i}$ in the general Hermitian solution $X=X_{1}+X_{2} i+X_{3} j+X_{4} k$ to (1.1) are given by

$$
\begin{gathered}
\max _{X_{i} \in J_{i}} r\left(X_{i}\right)=\min \left\{n, r\left[\begin{array}{ccc}
0 & 0 & \bar{A}_{i} \\
\widetilde{A}_{i} & \phi(B) & \phi(C)
\end{array}\right]+2 n-4 r(A)-4 r(G)\right\} ; \\
\min _{X_{i} \in J_{i}} r\left(X_{i}\right)=2 r\left[\begin{array}{ccc}
0 & 0 & \bar{A}_{i} \\
\widetilde{A}_{i} & \phi(B) & \phi(C)
\end{array}\right]-r\left[\begin{array}{ccc}
0 & 0 & \bar{A}_{i} \\
0 & 0 & \phi^{T}(B) \\
\widetilde{A}_{i} & \phi(B) & \phi(C)
\end{array}\right] \\
-2 r\left[\begin{array}{ccc}
-A_{2} & -A_{3} & -A_{4} \\
A_{1} & -A_{4} & A_{3} \\
A_{4} & A_{1} & -A_{2} \\
-A_{3} & A_{2} & A_{1}
\end{array}\right],
\end{gathered}
$$

where

$$
\widetilde{A}_{1}=\left[\begin{array}{ccc}
A_{2} & A_{3} & -A_{4} \\
-A_{1} & -A_{4} & -A_{3} \\
A_{4} & -A_{1} & A_{2} \\
A_{3} & -A_{2} & -A_{1}
\end{array}\right], \widetilde{A}_{2}=\left[\begin{array}{ccc}
-A_{1} & A_{3} & -A_{4} \\
-A_{2} & -A_{4} & -A_{3} \\
-A_{3} & -A_{1} & A_{2} \\
A_{4} & -A_{2} & -A_{1}
\end{array}\right]
$$




$$
\begin{gathered}
\widetilde{A}_{3}=\left[\begin{array}{ccc}
-A_{1} & A_{2} & -A_{4} \\
-A_{2} & -A_{1} & -A_{3} \\
-A_{3} & A_{4} & A_{2} \\
A_{4} & A_{3} & -A_{1}
\end{array}\right], \widetilde{A}_{4}=\left[\begin{array}{ccc}
-A_{1} & A_{2} & A_{3} \\
-A_{2} & -A_{1} & -A_{4} \\
-A_{3} & A_{4} & -A_{1} \\
A_{4} & A_{3} & -A_{2}
\end{array}\right], \\
\bar{A}_{1}=\left[\begin{array}{ccc}
-A_{2} & A_{3} & A_{4} \\
-A_{1} & -A_{4} & -A_{3} \\
-A_{4} & -A_{1} & -A_{2} \\
A_{3} & A_{2} & -A_{1}
\end{array}\right], \bar{A}_{2}=\left[\begin{array}{ccc}
-A_{1} & A_{3} & A_{4} \\
A_{2} & -A_{4} & -A_{3} \\
-A_{3} & -A_{1} & -A_{2} \\
A_{4} & A_{2} & -A_{1}
\end{array}\right]^{T}, \\
\bar{A}_{3}=\left[\begin{array}{ccc}
-A_{1} & -A_{2} & A_{4} \\
A_{2} & -A_{1} & -A_{3} \\
-A_{3} & -A_{4} & -A_{2} \\
-A_{4} & A_{3} & -A_{1}
\end{array}\right]^{T}, \bar{A}_{4}=\left[\begin{array}{ccc}
-A_{1} & -A_{2} & A_{3} \\
A_{2} & -A_{1} & A_{4} \\
-A_{3} & -A_{4} & -A_{1} \\
-A_{4} & A_{3} & A_{2}
\end{array}\right]^{T}
\end{gathered}
$$

(b) The maximal and minimal ranks of $Y_{j}$ in a Hermitian solution $Y_{1}+Y_{2} i+$ $Y_{3} j+Y_{4} k$ to (1.1) are given by

$$
\begin{gathered}
\max _{Y_{j} \in T_{j}} r\left(Y_{j}\right)=\min \left\{p, r\left[\begin{array}{ccc}
0 & 0 & \bar{B}_{j} \\
\widetilde{B}_{j} & \phi(A) & \phi(C)
\end{array}\right]+2 p-4 r(B)-4 r(G)\right\} ; \\
\min _{Y_{j} \in T_{j}} r\left(Y_{j}\right)=2 r\left[\begin{array}{ccc}
0 & 0 & \bar{B}_{j} \\
\widetilde{B}_{j} & \phi(A) & \phi(C)
\end{array}\right]-r\left[\begin{array}{ccc}
0 & 0 & \bar{B}_{j} \\
0 & 0 & \phi^{T}(A) \\
\widetilde{B}_{j} & \phi(A) & \phi(C)
\end{array}\right] \\
-2 r\left[\begin{array}{ccc}
-B_{2} & -B_{3} & -B_{4} \\
B_{1} & -B_{4} & B_{3} \\
B_{4} & B_{1} & -B_{2} \\
-B_{3} & B_{2} & B_{1}
\end{array}\right],
\end{gathered}
$$

where

$$
\begin{aligned}
& \widetilde{B}_{1}=\left[\begin{array}{ccc}
B_{2} & B_{3} & -B_{4} \\
-B_{1} & -B_{4} & -B_{3} \\
B_{4} & -B_{1} & B_{2} \\
B_{3} & -B_{2} & -B_{1}
\end{array}\right], \widetilde{B}_{2}=\left[\begin{array}{ccc}
-B_{1} & B_{3} & -B_{4} \\
-B_{2} & -B_{4} & -B_{3} \\
-B_{3} & -B_{1} & B_{2} \\
B_{4} & -B_{2} & -B_{1}
\end{array}\right], \\
& \widetilde{B}_{3}=\left[\begin{array}{ccc}
-B_{1} & B_{2} & -B_{4} \\
-B_{2} & -B_{1} & -B_{3} \\
-B_{3} & B_{4} & B_{2} \\
B_{4} & B_{3} & -B_{1}
\end{array}\right], \widetilde{B}_{4}=\left[\begin{array}{ccc}
-B_{1} & B_{2} & B_{3} \\
-B_{2} & -B_{1} & -B_{4} \\
-B_{3} & B_{4} & -B_{1} \\
B_{4} & B_{3} & -B_{2}
\end{array}\right],
\end{aligned}
$$




$$
\begin{aligned}
& \bar{B}_{1}=\left[\begin{array}{ccc}
-B_{2} & B_{3} & B_{4} \\
-B_{1} & -B_{4} & -B_{3} \\
-B_{4} & -B_{1} & -B_{2} \\
B_{3} & B_{2} & -B_{1}
\end{array}\right]^{T}, \bar{B}_{2}=\left[\begin{array}{ccc}
-B_{1} & B_{3} & B_{4} \\
B_{2} & -B_{4} & -B_{3} \\
-B_{3} & -B_{1} & -B_{2} \\
-B_{4} & B_{2} & -B_{1}
\end{array}\right]^{T}, \\
& \bar{B}_{3}=\left[\begin{array}{ccc}
-B_{1} & -B_{2} & B_{4} \\
B_{2} & -B_{1} & -B_{3} \\
-B_{3} & -B_{4} & -B_{2} \\
-B_{4} & B_{3} & -B_{1}
\end{array}\right]^{T}, \bar{B}_{4}=\left[\begin{array}{ccc}
-B_{1} & -B_{2} & B_{3} \\
B_{2} & -B_{1} & B_{4} \\
-B_{3} & -B_{4} & -B_{1} \\
-B_{4} & B_{3} & B_{2}
\end{array}\right]^{T} .
\end{aligned}
$$

Proof. We only derive the maximal and minimal ranks of the matrix $X_{1}$. The others can be established similarly. Applying Lemma 2.3 to (3.5), we get the following

$$
\begin{aligned}
\max _{X_{1} \in J_{1}} r\left(X_{1}\right) & =\max _{Z=Z^{T}, V} r\left(M+\frac{1}{4} \hat{P} Z \hat{P}^{T}+P V+V^{T} P^{T}\right) \\
& =\min \left\{n, \max _{Z=Z^{T}} r(U)\right\} \\
& =\min \left\{n, r\left[\begin{array}{ccc}
M & P & \hat{P} \\
P^{T} & 0 & 0
\end{array}\right]\right\}, \\
\min _{X_{1} \in J_{1}} r\left(X_{1}\right) & =\min _{Z=Z^{T}, V} r\left(M+\frac{1}{4} \hat{P} Z \hat{P}^{T}+P V+V^{T} P^{T}\right) \\
& =\min _{Z=Z^{T}} r(U)-2 r(P) \\
& =2 r\left[\begin{array}{ccc}
M & P & \hat{P} \\
P^{T} & 0 & 0
\end{array}\right]-r\left[\begin{array}{ccc}
M & P & \hat{P} \\
P^{T} & 0 & 0 \\
\hat{P}^{T} & 0 & 0
\end{array}\right]-2 r(P),
\end{aligned}
$$

where

$$
\begin{aligned}
U & =\left[\begin{array}{cc}
M+\frac{1}{4} \hat{P} Z \hat{P}^{T} & P \\
P^{T} & 0
\end{array}\right]=\left[\begin{array}{cc}
M & P \\
P^{T} & 0
\end{array}\right]+\frac{1}{4}\left[\begin{array}{l}
\hat{P} \\
0
\end{array}\right] Z\left[\begin{array}{cc}
P^{T} & 0
\end{array}\right], \\
M & =\frac{1}{4} P_{1} \phi\left(X_{0}\right) P_{1}^{T}+\frac{1}{4} P_{2} \phi\left(X_{0}\right) P_{2}^{T}+\frac{1}{4} P_{3} \phi\left(X_{0}\right) P_{3}^{T}+\frac{1}{4} P_{4} \phi\left(X_{0}\right) P_{4}^{T}, \\
P & =\left[P_{1}, P_{2}, P_{3}, P_{4}\right] L_{\phi(A)}, \hat{P}=\left[P_{1}, P_{2}, P_{3}, P_{4}\right] E_{1} .
\end{aligned}
$$


By Lemma 2.4, block Gaussian elimination, and $A X_{0} A^{*}+B Y_{0} B^{*}=C$, we have that

$$
\begin{aligned}
r\left[\begin{array}{ccc}
M & P & \hat{P} \\
P^{T} & 0 & 0
\end{array}\right] & =r\left[\begin{array}{cccc}
M & D_{1} & D_{2} & 0 \\
D_{1}^{T} & 0 & 0 & \Psi\left(A^{*}\right) \\
0 & \Psi(A) & 0 & 0 \\
0 & 0 & \Psi(G) & 0
\end{array}\right]-4 r(\phi(A)) \\
& -4 r\left(\phi\left(A^{*}\right)\right)-4 r(\phi(G)) \\
& =r\left[\begin{array}{ccc}
0 & 0 & \bar{A}_{1} \\
\widetilde{A}_{1} & \phi(B) & \phi(C)
\end{array}\right]+2 n-4 r(A)-4 r(G),
\end{aligned}
$$

where

$$
\begin{aligned}
D_{1} & =\left[P_{1}, P_{2}, P_{3}, P_{4}\right], D_{2}=\left[P_{1}, P_{2}, P_{3}, P_{4}\right] \phi\left(S_{1}\right), \\
\Psi(V) & =\left[\begin{array}{cccc}
\phi(V) & 0 & 0 & 0 \\
0 & \phi(V) & 0 & 0 \\
0 & 0 & \phi(V) & 0 \\
0 & 0 & 0 & \phi(V)
\end{array}\right], V=A, A^{*}, G .
\end{aligned}
$$

Similarly, we can simplify the following

$$
\begin{aligned}
& r\left[\begin{array}{ccc}
M & P & \hat{P} \\
P^{T} & 0 & 0 \\
\hat{P}^{T} & 0 & 0
\end{array}\right]=r\left[\begin{array}{ccc}
0 & 0 & \bar{A}_{1} \\
0 & 0 & \phi^{T}(B) \\
\widetilde{A}_{1} & \phi(B) & \phi(C)
\end{array}\right]+2 n-4 r(G)-4 r\left(G^{*}\right), \\
& r(P)=r\left[\begin{array}{ccc}
-A_{2} & -A_{3} & -A_{4} \\
A_{1} & -A_{4} & A_{3} \\
A_{4} & A_{1} & -A_{2} \\
-A_{3} & A_{2} & A_{1}
\end{array}\right]+n-4 r(A)
\end{aligned}
$$

Thus we have the results for extreme ranks of the matrix $X_{1}$ in (a). Similarly, applying Lemma 2.3 and Lemma 2.4 to (3.6)-(3.12) yields the other results in (a) and (b).

Corollary 3.3. Suppose that the matrix equation (1.1) has a Hermitian solution over $\mathbb{H}$, then we have the following:

(a) (1.1) has a real symmetric solution $X$ if and only if, for $i=2,3,4$,

$$
\begin{aligned}
2 r\left[\begin{array}{ccc}
0 & 0 & \bar{A}_{i} \\
\widetilde{A}_{i} & \phi(B) & \phi(C)
\end{array}\right] & =r\left[\begin{array}{ccc}
0 & 0 & \bar{A}_{i} \\
0 & 0 & \phi^{T}(B) \\
\widetilde{A}_{i} & \phi(B) & \phi(C)
\end{array}\right] \\
+2 r & {\left[\begin{array}{ccc}
-A_{2} & -A_{3} & -A_{4} \\
A_{1} & -A_{4} & A_{3} \\
A_{4} & A_{1} & -A_{2} \\
-A_{3} & A_{2} & A_{1}
\end{array}\right] . }
\end{aligned}
$$


In that case, the real symmetric solution $X$ can be expressed as $X=X_{1}$ in (3.5).

(b) All the solutions of (1.1) for $X$ are real symmetric if and only if

$$
r\left[\begin{array}{ccc}
0 & 0 & \bar{A}_{i} \\
\widetilde{A}_{i} & \phi(B) & \phi(C)
\end{array}\right]+2 n=4 r(A)+4 r(G), i=2,3,4 .
$$

In that case, the real symmetric solution $X$ can be expressed as $X=X_{1}$ in (3.5).

(c) (1.1) has a complex Hermitian solution $X$ if and only if, for $i=3,4$,

$$
\begin{aligned}
2 r\left[\begin{array}{ccc}
0 & 0 & \bar{A}_{i} \\
\widetilde{A}_{i} & \phi(B) & \phi(C)
\end{array}\right] & =r\left[\begin{array}{ccc}
0 & 0 & \bar{A}_{i} \\
0 & 0 & \phi^{T}(B) \\
\widetilde{A}_{i} & \phi(B) & \phi(C)
\end{array}\right] \\
& +2 r\left[\begin{array}{ccc}
-A_{2} & -A_{3} & -A_{4} \\
A_{1} & -A_{4} & A_{3} \\
A_{4} & A_{1} & -A_{2} \\
-A_{3} & A_{2} & A_{1}
\end{array}\right] .
\end{aligned}
$$

In that case, the complex Hermitian solution $X$ can be expressed as $X=$ $X_{1}+X_{2} i$, where $X_{1}, X_{2}$ are expressed as (3.5) and (3.6).

(d) All the solutions of (1.1) for $X$ are complex Hermitian if and only if

$$
r\left[\begin{array}{ccc}
0 & 0 & \bar{A}_{i} \\
\widetilde{A}_{i} & \phi(B) & \phi(C)
\end{array}\right]+2 n=4 r(A)+4 r(G), i=3,4
$$

In that case, the complex Hermitian solution $X$ can be expressed as $X=$ $X_{1}+X_{2} i$, where $X_{1}, X_{2}$ are expressed as (3.5) and (3.6).

(e) (1.1) has a pure imaginary Hermitian solution $X$ if and only if

$$
\begin{aligned}
2 r\left[\begin{array}{ccc}
0 & 0 & \bar{A}_{1} \\
\widetilde{A}_{1} & \phi(B) & \phi(C)
\end{array}\right]=r\left[\begin{array}{ccc}
0 & 0 & \bar{A}_{1} \\
0 & 0 & \phi^{T}(B) \\
\widetilde{A}_{1} & \phi(B) & \phi(C)
\end{array}\right] \\
+2 r\left[\begin{array}{ccc}
-A_{2} & -A_{3} & -A_{4} \\
A_{1} & -A_{4} & A_{3} \\
A_{4} & A_{1} & -A_{2} \\
-A_{3} & A_{2} & A_{1}
\end{array}\right] .
\end{aligned}
$$

In that case, the pure imaginary Hermitian solution $X$ can be expressed as $X=X_{2} i+X_{3} j+X_{4} k$, where $X_{2}, X_{3}$, and $X_{4}$ are expressed as (3.6), (3.7), and (3.8). 
(f) All the solutions of (1.1) for $X$ are pure imaginary Hermitian if and only if

$$
r\left[\begin{array}{ccc}
0 & 0 & \bar{A}_{1} \\
\widetilde{A}_{1} & \phi(B) & \phi(C)
\end{array}\right]+2 n=4 r(A)+4 r(G) .
$$

In that case, the pure imaginary Hermitian solution $X$ can be expressed as $X=X_{2} i+X_{3} j+X_{4} k$, where $X_{2}, X_{3}$, and $X_{4}$ are expressed as (3.6), (3.7), and (3.8).

Using the same method, we can get the corresponding results on $Y$.

REMARK 3.4. Similarly, we can get the corresponding results on the skewHermitian solution of (1.1).

4. Ranks of Hermitian solution to some special cases of (1.1). In this section, we consider some special cases of (1.1) over $\mathbb{C}$. When $B$ vanishes, (1.1) becomes (1.2) where $A \in \mathbb{C}^{m \times n}, C \in \mathbb{C}^{m \times m}$. We get the corresponding results on (1.2) as follows.

Corollary 4.1. Let $A=A_{1}+A_{2} i \in \mathbb{C}^{m \times n}, C=C^{*}=C_{1}+C_{2} i \in \mathbb{C}^{m \times m}$ be given. Then

(a) The matrix equation (1.2) has a Hermitian solution if and only if the real matrix equation

$$
\left[\begin{array}{cc}
A_{1} & -A_{2} \\
A_{2} & A_{1}
\end{array}\right]\left[\begin{array}{ll}
X_{11} & X_{12} \\
X_{21} & X_{22}
\end{array}\right]\left[\begin{array}{cc}
A_{1}^{T} & A_{2}^{T} \\
-A_{2}^{T} & A_{1}^{T}
\end{array}\right]=\left[\begin{array}{cc}
C_{1} & -C_{2} \\
C_{2} & C_{1}
\end{array}\right]
$$

has a symmetric solution over $\mathbb{R}$. In this case, the general Hermitian solution of (1.2) over $\mathbb{C}$ can be written as

$$
X=X_{1}+X_{2} i=\frac{1}{2}\left(X_{11}+X_{22}\right)+\frac{1}{2}\left(X_{12}^{T}-X_{12}\right) i,
$$

where $X_{t t}=X_{t t}^{T}, t=1,2 ;$ and $X_{12}^{T}=X_{21}$ are the general solutions of (4.1) over $\mathbb{R}$. Written in an explicit form, $X_{1}, X_{2}$ in (4.2) are

$$
\begin{aligned}
X_{1} & =\frac{1}{2} P_{1} \phi\left(X_{0}\right) P_{1}^{T}+\frac{1}{2} P_{2} \phi\left(X_{0}\right) P_{2}^{T}+\left[P_{1}, P_{2}\right] L_{\phi(A)}\left[\begin{array}{c}
V_{1} \\
V_{2}
\end{array}\right] \\
& +\left[\begin{array}{c}
V_{1} \\
V_{2}
\end{array}\right]^{T} L_{\phi(A)}^{T}\left[P_{1}, P_{2}\right]^{T}, \\
X_{2} & =\frac{1}{2} P_{2} \phi\left(X_{0}\right) P_{1}^{T}-\frac{1}{2} P_{1} \phi\left(X_{0}\right) P_{2}^{T}-\left[-P_{2}, P_{1}\right] L_{\phi(A)}\left[\begin{array}{c}
V_{1} \\
V_{2}
\end{array}\right] \\
& +\left[\begin{array}{c}
V_{1} \\
V_{2}
\end{array}\right]^{T} L_{\phi(A)}^{T}\left[-P_{2}, P_{1}\right]^{T},
\end{aligned}
$$


where $X_{0}=A^{\dagger} C\left(A^{\dagger}\right)^{*}, P_{1}=\left[I_{n}, 0\right], P_{2}=\left[0, I_{n}\right]$, and $V_{1}$ and $V_{2}$ are arbitrary real matrices with compatible sizes.

(b) Put

$$
\begin{aligned}
& J_{1}=\left\{X_{1} \in \mathbb{R}^{n \times n} \mid A\left(X_{1}+X_{2} i\right) A^{*}=C\right\}, \\
& J_{2}=\left\{X_{2} \in \mathbb{R}^{n \times n} \mid A\left(X_{1}+X_{2} i\right) A^{*}=C\right\} .
\end{aligned}
$$

Then we have the following:

(i) The maximal and minimal ranks of $X_{1}$ in the Hermitian solution $X=$ $X_{1}+X_{2} i$ to (1.2) are given by

$$
\begin{aligned}
& \max _{X_{1} \in J_{1}} r\left(X_{1}\right)=\min \left\{n, r\left[\begin{array}{ccc}
C_{1} & -C_{2} & A_{1} \\
C_{2} & C_{1} & A_{2} \\
A_{1}^{T} & A_{2}^{T} & 0
\end{array}\right]+2 n-4 r(A)\right\}, \\
& \min _{X_{1} \in J_{1}} r\left(X_{1}\right)=r\left[\begin{array}{ccc}
C_{1} & -C_{2} & A_{1} \\
C_{2} & C_{1} & A_{2} \\
A_{1}^{T} & A_{2}^{T} & 0
\end{array}\right]-2 r\left[\begin{array}{l}
A_{1} \\
A_{2}
\end{array}\right] .
\end{aligned}
$$

(ii) The maximal and minimal ranks of $X_{2}$ in the Hermitian solution $X=$ $X_{1}+X_{2} i$ to (1.2) are given by

$$
\begin{aligned}
& \max _{X_{2} \in J_{2}} r\left(X_{2}\right)=\min \left\{n, r\left[\begin{array}{ccc}
C_{1} & -C_{2} & A_{1} \\
C_{2} & C_{1} & A_{2} \\
A_{2}^{T} & -A_{1}^{T} & 0
\end{array}\right]+2 n-4 r(A)\right\}, \\
& \min _{X_{2} \in J_{2}} r\left(X_{2}\right)=r\left[\begin{array}{ccc}
C_{1} & -C_{2} & A_{1} \\
C_{2} & C_{1} & A_{2} \\
A_{2}^{T} & -A_{1}^{T} & 0
\end{array}\right]-2 r\left[\begin{array}{l}
A_{1} \\
A_{2}
\end{array}\right] .
\end{aligned}
$$

Remark 4.2. Corollary 4.1 is Theorem 2.2 of [13]. Similarly, Theorem 3.2 of [13] can be regarded as a special case of (1.1) with skew-Hermitian solutions.

Acknowledgment. The authors would like to thank Professor Michael Neumann and a referee very much for their valuable suggestions and comments, which resulted in a great improvement of the original manuscript.

\section{REFERENCES}

[1] J.K. Baksalary. Nonnegative definite and positive definite solutions to the matrix equation $A X A^{*}=B$. Linear and Multilinear Algebra, 16:133-139, 1984.

[2] X.W. Chang and J.S. Wang. The symmetric solution of the matrix equations $A X+Y A=C$, $A X A^{T}+B Y B^{T}=C$, and $\left(A^{T} X A, B^{T} X B\right)=(C, D)$. Linear Algebra Appl., 179:171-189, 1993. 
[3] H. Dai and P. Lancaster. Linear matrix equations from an inverse problem of vibration theory. Linear Algebra Appl., 246:31-47, 1996.

[4] M. Dehghan and M. Hajarian. An iterative algorithm for the reflexive solutions of the generalized coupled Sylvester matrix equations and its optimal approximation. Appl. Math. Comput., 202:571-588, 2008.

[5] M. Dehghan and M. Hajarian. Finite iterative algorithms for the reflexive and anti-reflexive solutions of the matrix equation $A_{1} X_{1} B_{1}+A_{2} X_{2} B_{2}=C$. Math. Comput. Modelling, 49: 1937-1959, 2009.

[6] M. Dehghan and M. Hajarian. On the reflexive solutions of the matrix equation $A X B+C Y D=$ E. Bull. Korean Math. Soc., 46: 511-519, 2009.

[7] J. Groß. A note on the general Hermitian solution to $A X A^{*}=$ B. Bull. Malaysian Math. Soc., 21:57-62, 1998 .

[8] J. Groß. Nonnegtive-definite and positive-definite solution to the matrix equation $A X A^{*}=B$ revisited. Linear Algebra Appl., 321:123-129, 2000.

[9] T.W. Hungerford. Algebra. Spring-Verlag Inc., New York, 1980.

[10] Y.T. Li and W.J. Wu. Symmetric and skew-antisymmetric solutions to systems of real quaternion matrix equations. Comput. Math. Appl., 55:1142-1147, 2008.

[11] A.P. Liao and Z.Z. Bai. The constrained solutions of two matrix equations. Acta Math. Sin., (Engl. Ser.), 18:671-678, 2002.

[12] Y. Liu and Y. Tian. More on extremal ranks of the matrix expressions $A-B X \pm X^{*} B^{*}$ with statistical applications. Numer. Linear Algebra Appl., 15:307-325, 2008.

[13] Y. Liu, Y. Tian, and Y. Takane. Ranks of Hermitian and skew-Hermitian solutions to the matrix equation $A X A^{*}=B$. Linear Algebra Appl., 431:2359-2372, 2009.

[14] G. Marsaglia and G. Styan. Equalities and inequalities for ranks of matrices. Linear Multilinear Algebra, 2:269-292, 1974.

[15] S.K. Mitra. The matrix equations $A X=C, X B=D$. Linear Algebra Appl., 59:171-181, 1984.

[16] S.K. Mitra. A pair of simultaneous linear matrix equations $A_{1} X B_{1}=C_{1}, A_{2} X B_{2}=C_{2}$ and a programming problem. Linear Algebra Appl., 131:107-123, 1990.

[17] J. B. Rosen. Minimum and basic solutions to singular linear systems. J. Soc. Indust. Appl. Math., 12:15-162, 1964.

[18] Y. Tian. The solvability of two linear matrix equations. Linear Multilinear Algebra, 48:123-147, 2000.

[19] Y. Tian. Ranks of Solutions of the matrix equation $A X B=$ C. Linear Multilinear Algebra, 51:111-125, 2003.

[20] Y. Tian. Ranks and independence of solutions of the matrix equation $A X B+C Y D=M$. Acta Math. Univ. Comenian. (N.S.), 1:75-84, 2006.

[21] Y. Tian and Y. Liu. Extremal ranks of some symmetric matrix expressions with applications. SIAM J. Matrix Anal. Appl., 28:890-905, 2006.

[22] F. Uhlig. On the matrix equation $A X=B$ with applications to the generators of controllability matrix. Linear Algebra and Appl., 85:203-209, 1987.

[23] Q.W. Wang. A system of matrix equations and a linear matrix equation over arbitrary regular rings with identity. Linear Algebra Appl., 384:43-54, 2004.

[24] Q.W. Wang. A system of four matrix equations over von Neumann regular rings and its applications. Acta Math. Sin. (Engl. Ser.), 21:323-334, 2005.

[25] Q.W. Wang. Bisymmetric and centrosymmetric solutions to systems of real quaternion matrix equations. Comput. Math. Appl., 49:641-650, 2005.

[26] Q.W. Wang. The general solution to a system of real quaternion matrix equations. Comput. Math. Appl., 49:665-675, 2005.

[27] Q.W. Wang and C.K. Li. Ranks and the least-norm of the general solution to a system of quaternion matrix equations. Linear Algebra Appl., 430:1626-1640, 2009. 
[28] Q.W. Wang, G.J. Song, and C.Y. Lin. Extreme ranks of the solution to a consistent system of linear quaternion matrix equations with an application. Appl. Math. Comput., 189:1517$1532,2007$.

[29] Q.W. Wang, J.H. Sun and S.Z. Li. Consistency for bi(skew)symmetric solutions to systems of generalized Sylvester equations over a finite central algebra. Linear Algebra Appl., 353:169-182, 2002.

[30] Q.W. Wang, Y.G. Tian and S.Z. Li. Roth's theorems for centroselfconjugate solutions to systems of matrix equations over a finite dimensional central algebra. Southeast Asian Bull. Math., 27:929-938, 2004.

[31] Q.W. Wang, J.W. van der Woude, and H.X. Chang. A system of real quaternion matrix equations with applications. Linear Algebra Appl., 431:2291-2303, 2009.

[32] Q.W. Wang and F. Zhang. The reflexive re-nonnegative definite solution to a quaternion matrix equation. Electron. J. Linear Algebra, 17:88-101, 2008.

[33] Q.W. Wang, H.S. Zhang, and G.J. Song. A new solvable condition for a pair of generalized Sylvester equations. Electron. J. Linear Algebra, 18:289-301, 2009.

[34] Q.W. Wang, H.S. Zhang, and S.W. Yu. On solutions to the quaternion matrix equation $A X B+$ $C Y D=$ E. Electron. J. Linear Algebra, 17:343-358, 2008.

[35] Q.W. Wang, Y. Zhou, and Q. Zhang. Ranks of the common solution to six quaternion matrix equations. Acta Math. Appl. Sin. Engl. Ser., DOI:10.1007/s10255-009-8168-4.

[36] M. Wei and Q. Wang. On rank-constrained Hermitian nonnegative-definite least squares solutions to the matrix equation $A X A^{*}=$ B. Int. J. Comput. Math., 84:945-952, 2007.

[37] D. Xie, Z. Zhang, and Z. Liu. Theory and method for updating least-squares finite element model of symmetric generalized centro-symmetric matrices. J. Comput. Appl. Math., 216:484-497, 2008.

[38] G. Xu, M. Wei, and D. Zheng. On solution of matrix equation $A X B+C Y D=F$. Linear Algebra Appl., 279:93-109, 1998.

[39] S. Yuan, A. Liao, and Y. Lei. Least squares Hermitian solution of the matrix equation $(A X B, C X D)=(E, F)$ with the least norm over the skew field of quaternions. Math. Comput. Modelling, 48:91-100, 2008.

[40] X. Zhang. The general Hermitian nonnegative-definite solution to the matrix equation $A X A^{*}+$ $B Y B^{*}=$ C. Acta Math. Acad. Paedagog. Nyhazi. (N.S.), 21:33-42, 2005.

[41] X. Zhang and M. Cheng. The rank-constrained Hermitian nonnegtive-definite and positivedefinite solutions to the matrix equation $A X A^{*}=B$. Linear Algebra Appl., 370:163-174, 2003.

[42] B. Zheng, L. Ye, and Dragana S. Cvetković-Ilić. The * congruence class of the solutions of some matrix equations. Comput. Math. Appl., 57:540-549, 2009. 